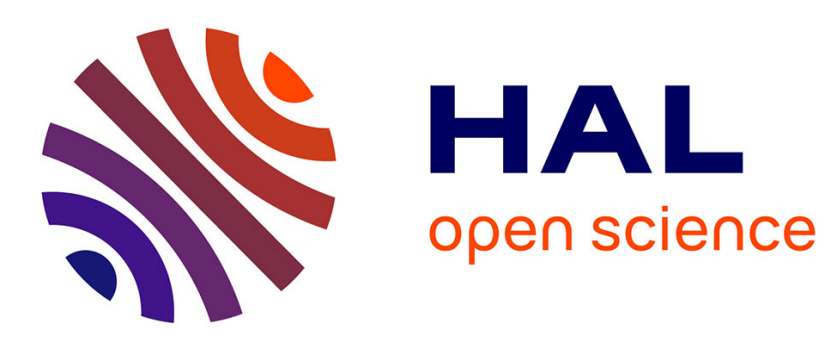

\title{
Insect inspired autopilots
}

Nicolas Franceschini, Franck Ruffier, Julien Serres

\section{To cite this version:}

Nicolas Franceschini, Franck Ruffier, Julien Serres. Insect inspired autopilots. The International Symposium on Aero-aqua Bio-Mechanisms (ISABMEC), Aug 2009, Shangai, China. hal-02195500

\section{HAL Id: hal-02195500 \\ https://hal-amu.archives-ouvertes.fr/hal-02195500}

Submitted on 26 Jul 2019

HAL is a multi-disciplinary open access archive for the deposit and dissemination of scientific research documents, whether they are published or not. The documents may come from teaching and research institutions in France or abroad, or from public or private research centers.
L'archive ouverte pluridisciplinaire HAL, est destinée au dépôt et à la diffusion de documents scientifiques de niveau recherche, publiés ou non, émanant des établissements d'enseignement et de recherche français ou étrangers, des laboratoires publics ou privés.

\section{(c)(1)}

Distributed under a Creative Commons Attribution| 4.0 International License 


\section{Insect inspired autopilots}

\author{
N. Franceschini*, F. Ruffier and J. Serres \\ Institute of Movement Science, Biorobotics Lab \\ CNRS and University of Aix-Marseille, France
}

Abstract: We address some of the control problems involved in insects' and robots' visually guided piloting. We present explicit control schemes that explain how insects may navigate on the basis of optic flow (OF) cues, without requiring any distance and speed measurements. The concept of the optic flow regulator, a feedback control system based on OF sensors, is presented. We tested our control schemes in simulation, and implemented them onboard two types of aerial robots, a helicopter and a hovercraft. Our electronic OF sensors were inspired by the results of our microelectrode studies on motion sensitive neurons in the housefly's compound eye. The control schemes described do not involve any conventional avionic sensors like rangefinders or speed sensors, and show great potential for the autonomous control of air, underwater and space vehicles.

Index terms: Visuo-motor control, optic flow, Insect, Micro-air vehicles (MAV)

\section{INTRODUCTION}

Conventional aircraft autopilots require the measurement of state variables such as barometric altitude, groundheight, groundspeed, descent speed, etc. The sensors developed for this purpose - usually emissive sensors such as radar-altimeters, forward-looking infrared sensors, Doppler radars, GPS, etc. - are far too cumbersome for insects or even birds to carry and to power. Natural flyers have developed other systems for controlling their flight and they can teach us some lessons. Flying insects are agile creatures navigating swiftly through most unpredictable environments. Equipped with "only" about one million neurons and only 3000 pixels in each eye, the housefly, for example, achieves 3D navigation at an impressive 700 bodylengths per second. This objectionable creature actually achieves just what is being sought for in the field of aerial robotics: dynamic stabilization, 3D autonomous piloting, ground avoidance, collision avoidance with stationary and nonstationary obstacles, tracking, docking, autonomous takeoff and landing, etc. The last seven decades have provided evidence that flying insects guide themselves through their environments by processing the optic flow (OF) that is generated on their eyes as a consequence of their locomotion. In an animal's reference frame, the translational $\mathrm{OF}$ is the angular speed $\omega$ at which contrasting objects in the environment move past the animal.

* Corresponding author: CNRS \& Aix-Marseille University, Institute of Movement Science, Biorobotics Lab, MARSEILLE-Luminy (France) nicolas.franceschini@univmed.fr
This contribution summarizes two recent reviews $[2,3]$, in which we recounted our attempts to model the visuomotor control system that provides flying insects with a means of autonomous piloting at close range. The interested reader is referred to these reviews and original papers for an extensive litterature on both insects' and robots' vision based autopilots. The aim of these studies was not to produce a detailed neural circuit of the visuomotor control systems, but rather to obtain a more functional overall picture, that is, a picture that abstracts some basic control principles.

Our progress on these lines was achieved by performing simulation experiments and testing our control schemes onboard miniature aircraft. These aerial robots are based on the use of electronic OF sensors inspired by the housefly Elementary Motion Detectors (EMDs), which we had previously analysed in our laboratory.

\section{FROM THE FLY COMPOUND EYE TO BIO- INSPIRED OPTIC FLOW SENSORS}

Each compound eye consists of an array of ommatidia, the frontend of which is a facet lens focussing light on a group of photoreceptor cells (Fig. 1). The fly retina has been described in great details, with the different spectral types of cells, polarization sensitive cells and female tracking cells in the male. There exists a

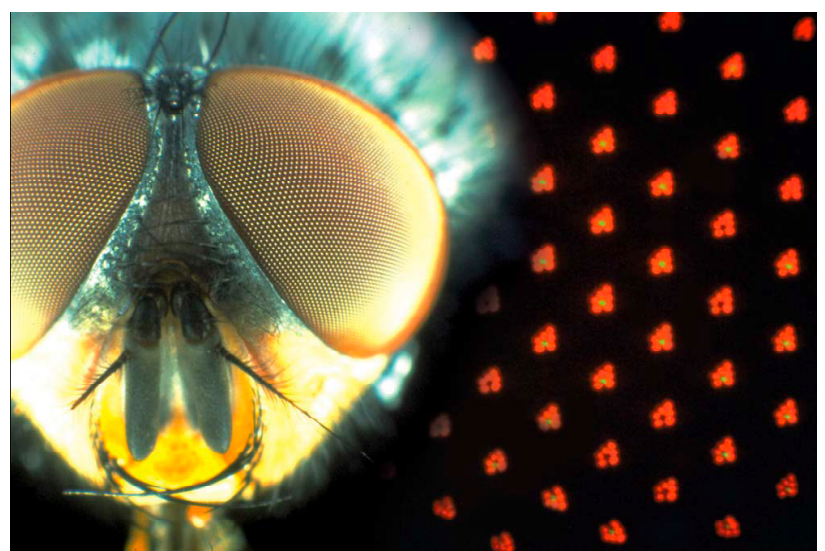

Figure 1: Head of a blowfly with its two panoramic compound eyes, and part of the receptor mosaic observed in vivo after optical neutralization of the cornea. Each micrometer-sized photoreceptor has a distinct autofluorescence color linked to its specific visual pigment (from [3]).

typical division of labour within the retina:

- The two central (tandem) photoreceptor cells, R7-8, display various spectral sensitivities that are randomly scattered across the retinal mosaic, as attested by the characteristic R7 autofluorescence colors (Fig. 1). $\mathrm{R} 7$ and $\mathrm{R} 8$ are thought to participate in color vision.

- The outer 6 photoreceptor cells (R1-R6) all have the same spectral sensitivity and participate, in particular, in motion detection. In this visual pathway, signal-to-noise ratio is improved by ingenuous features : (i) a UV sensitizing pigment that enhances the quantum catch, and (ii) an ingenuous opto-neural projection called "neural superposition". 
To estimate the OF, insects use motion sensitive neurons. In flies, part of the 3rd optic ganglion called the Lobula Plate (LP) appears as a genuine "visual motion processing center". It comprises approximately 60 uniquely identifiable neurons, the LP tangential cells (LPTC), that analyze the OF field resulting from the animal's walking or flying. Some of these neurons transmit their electrical signals via the neck to thoracic interneurons that will drive the wing-, leg-, and headmuscles. Other neurons send their signals to the contralateral eye. The LPTCs are known to be large-field collator neurons that pool the electrical signals from many retinotopic input elements called "Elementary Motion Detectors" (EMDs). Although the cellular details underlying a single EMD are still elusive, we analysed its functioning in the housefly, using microelectrode recording from an identified neuron combined with single photoreceptor stimulation. With a special optical instrument whose main objective was quite simply one facet lenslet (diameter $\cong 25 \mu \mathrm{m}$, focal length $\cong 50 \mu \mathrm{m}$ ), we illuminated two photoreceptors (diameter $\cong 1 \mu \mathrm{m}$ ) sequentially within the selected ommatidium. The H1neuron responded with a vigorous spike discharge to this "apparent motion", provided the motion was mimicked in the preferred direction, and did not respond to a motion mimicked in the non-preferred (null) direction. From many experiments of this kind, in which various sequences of light steps and/or pulses were applied to selected receptor pairs, we established an EMD block diagram and characterized each block's dynamics and nonlinearity. While not unveiling the cellular details of the EMD circuit, our analysis allowed the EMD principle to be understood functionally - opening the way to its transcription in electronics.

(a)

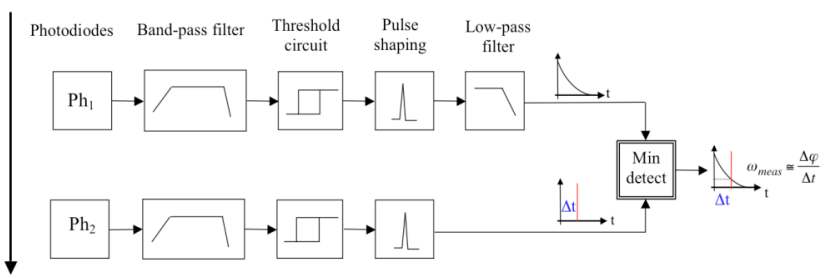

(b)
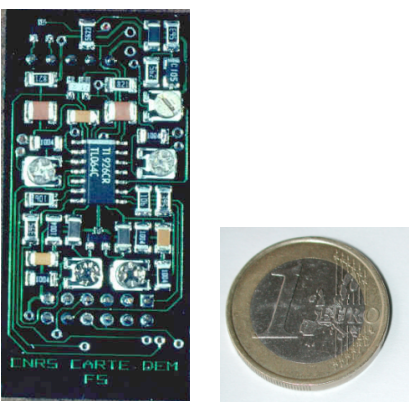

Figure 2: (a) Principle of the optic flow sensor inspired by the results of our electrophysiological analyses of the housefly's EMD. (b) purely analog version (mass : 5 grams) built in 1989 for the robot-Fly whose compound eye housed a ring of 114 EMDs of this type (c) hybrid (analog + digital) version (size : $7 \mathrm{~mm} \mathrm{x} 7 \mathrm{~mm}$, mass 0.2 grams) based on a microcontroller and built using Low Temperature Co-fired Ceramics technology (LTCC) (from [3]).
In the mid 1980's, we designed an optic flow sensor, the signal processing scheme of which (Fig. 2a) was inspired by lessons taken from the fly EMD. The OF is an angular speed $\omega$ [rad.s ${ }^{-1}$ ] equal to the inverse of the time $\Delta \mathrm{t}$ taken by a contrasting feature to travel between the visual axes of two adjacent photoreceptors, separated by an angle $\Delta \varphi$. Our OF sensor processes this time lapse $\Delta \mathrm{t}$ so as to generate a response that grows monotonically with the inverse of $\Delta t$, and hence with the optic flow $\omega$ (Fig. 2a). A short $\Delta \mathrm{t}$ gives a high voltage output and vice versa. The thresholding makes the response relatively independent of contrast and spatial frequency, unlike the Reichardt "correlator scheme" for motion detection.

\section{III. "OPTIC FLOW REGULATORS” AS VISION BASED AUTOPILOTS}

A

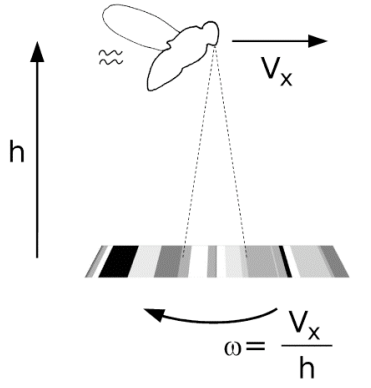

B
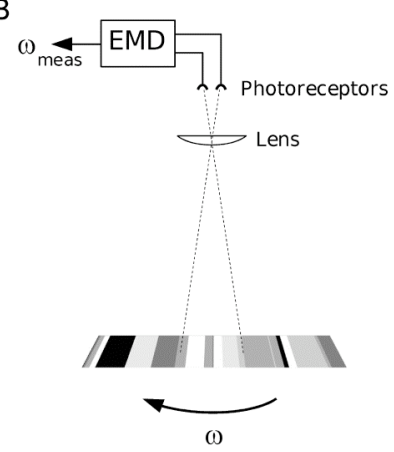

Figure 3: Definition (A) and measurement of the ventral optic flow $\boldsymbol{w}$ experienced by an insect (or a pilot, or a robot) flying in translation in the vertical plane. (B) an EMD of the type shown in Fig. 2 is able to measure the ventral OF, i.e., the angular speed $\boldsymbol{w}\left[\operatorname{rad}^{-1} \mathrm{~s}^{-1}\right.$, at which a contrasting feature moves under the flying agent (from [1]).

The ventral OF experienced in the longitudinal plane by flying creatures - including aircraft pilots - is the apparent angular velocity $\omega$ generated by a point directly below on the flight track. As shown in figure $3 \mathrm{~A}$, the ventral OF depends on both the groundspeed $\boldsymbol{V}_{\boldsymbol{x}}$ and the groundheight $\boldsymbol{h}$ and is equal to the ratio between these two variables:

$$
\boldsymbol{\omega}=\boldsymbol{V}_{\boldsymbol{x}} / \boldsymbol{h}\left[\mathrm{rad} . \mathrm{s}^{-1}\right]
$$

Flies and bees have been shown to react to the translational $O F$ independently of the spatial texture and contrast, and some of their visual neurons - the "velocity tuned neurons" - may be involved in this reaction because they respond monotonically to $\omega$ with little dependence on texture and contrast. Neurons facing downwards can therefore act as ventral $O F$ sensors, and thus assess the $\boldsymbol{V}_{\boldsymbol{x}} / \boldsymbol{h}$ ratio (Fig. 3).

Sixty years ago, Kennedy put forward an "optomotor theory" of insect flight, according to which flying insects maintain a "preferred retinal velocity" with respect to the ground below. In the meantime, many experiments have confirmed this view: both flies and bees maintain a 
constant OF with respect to the ground while cruising or landing. The big problem is how insects achieve this feat, since there is an infinitely large number of possible combinations of $\boldsymbol{V}_{\boldsymbol{x}}$ and $\boldsymbol{h}$ generating the same $\boldsymbol{V}_{\boldsymbol{x}} / \boldsymbol{h}$ ratio. Kennedy's "theory" terefore called for an explicit control scheme that would clarify: (i) the flight variables really involved, (ii) the sensors really required (iii) the dynamics of the various system components, (iv) the causal and dynamic links between the sensor(s) and the variable(s) to be controlled, (v) the points of application and the effects of the various disturbances that insects may experience (change in relief, headwind, etc.).

In 1999, we established via experimental simulation how a seeing helicopter (or an insect) might manage to follow a terrain and land on the sole basis of OF cues without measuring its groundspeed or groundheight (see Fig. 4,5 in [5]). The landing trajectory obtained in these simulations (Fig. 5 in [5]) resembled the final approach of bees landing on a flat surface. The 840-gram rotorcraft we constructed was able to jump over 1-meter high obstacles (see Fig. 8 in [6]).

We then developed a genuine "OF based autopilot" called OCTAVE (which stands for Optical Control sysTem for Aerial VEhicles), that enables a microhelicopter to perform challenging tasks $[7,1]$. The idea was to integrate an $\mathrm{OF}$ sensor into a feedback loop driving the robot's lift so as to compensate for any deviations of the OF sensor's output from a given setpoint (figure 4A). This is what we call the OF regulator for ground avoidance. The term "regulator" is used here as in control theory, to denote a feedback control system designed to maintain a variable (here the OF, $\omega)$ constantly equal to a given reference (the "set-point").

The OF sensor produces a signal $\omega_{\text {meas }}$ (figures $3 \mathrm{~B}$ ) that is compared with the OF set-point $\omega_{\text {Set }}$ (Fig. 4A). The error signal $\varepsilon=\omega_{\text {meas }}-\omega_{\text {Set }}$ drives a controller adjusting the lift $\boldsymbol{L}$, and hence the groundheight $\boldsymbol{h}$, so as to minimize $\varepsilon$ (Fig. 4A). All the operator does is to set the pitch angle $\Theta$, and hence the airspeed: the $O F$ regulator does the rest, holding the $\boldsymbol{V}_{\boldsymbol{x}}: \boldsymbol{h}$ ratio - i.e., the ventral OF - constant. In the steady state (i.e., at $t=\infty$ ), $\omega_{\text {meas }} \cong \omega_{\text {Set }}$ and the groundheight $\boldsymbol{h}$ becomes proportional to the groundspeed $\boldsymbol{V}_{\boldsymbol{x}}(E q .2)$ :

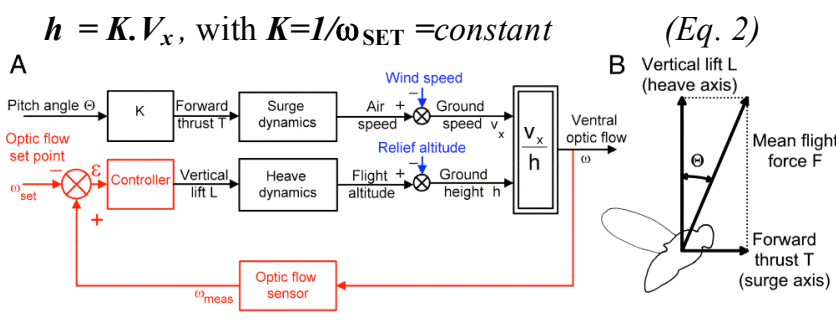

Figure 4: (A) The OCTAVE autopilot consists of a feedback control system, called the optic flow regulator (bottom part) that controls the vertical lift, and hence the groundheight, so as to maintain the ventral $\mathrm{OF}, \omega$, constant and equal to the set-point $\omega_{\text {set }}$ whatever the groundspeed $\boldsymbol{V}_{\boldsymbol{x}}$. (B) Like flies and bees, our micro-helicopter $(\mathrm{MH})$ gains speed by having its flight force vector $\boldsymbol{F}$ pichted forward at an angle $\Theta$ with respect to the vertical. Controlling $\boldsymbol{F}$ (via the rotor rpm) amounts to mainly controlling $\boldsymbol{L}$ because $\Theta$ always remains small ( $\boldsymbol{\Theta}_{\max }$ $<10^{\circ}$ for $\mathrm{V}_{\mathrm{x}} \max =3 \mathrm{~m} / \mathrm{s}$ ) (from [1]).
To test the robustness of this OF regulator scheme, we implemented it on a micro-helicopter $(\mathrm{MH})$ equipped with a two-pixel ventral eye driving a single EMD (Fig. $5 \mathrm{~A}$ ). The $\mathrm{MH}$ is tethered to the tip of a flight mill (Fig. 5B) equipped with ground-truth azimuthal and elevation sensors, with which the position and speed of the MH can be monitored accurately. Any increase in the rotor rpm causes the $\mathrm{MH}$ to lift and rise, and the slightest (operator mediated) forward pitching induces the $\mathrm{MH}$ to gain speed. The feedback reacts to this increase in forward speed $\boldsymbol{V}_{\boldsymbol{x}}$ by increasing the vertical lift $\boldsymbol{L}$ - and hence, the groundheight $\boldsymbol{h}$ - so as to hold the $\boldsymbol{V}_{\boldsymbol{x}} \boldsymbol{:} \boldsymbol{h}$ ratio constant.
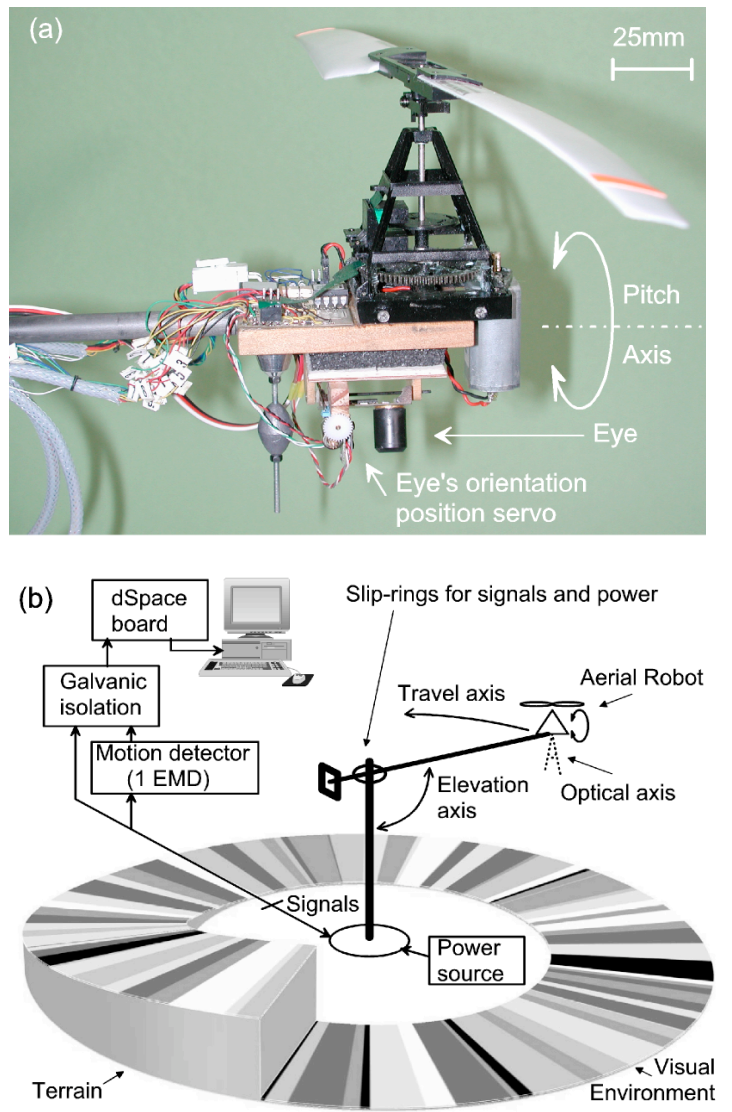

Figure $5:$ (a) 100-gram Micro-helicopter (MH) equipped with a ventral OF sensor (Fig. 2) and an $O F$ regulator (Fig. 4A) that acts upon the lift by altering the rotor rpm. (b) Tethered to the tip of the (pantographic) arm of the flight mill, the $\mathrm{MH}$ is remotely commanded to pitch forward at an angle $\Theta$. It makes successive laps over the arena (diameter $4.5 \mathrm{~m}$ ), the ground texture of which is randomly distributed in terms of both the spatial frequency and the contrast $\boldsymbol{m}(0.04<\boldsymbol{m}<0.3)$ (from [7]).

OCTAVE's $O F$ regulator scheme (Fig. 4A) results in the behavioral patterns shown in Fig. 6, which gives the $\mathrm{MH}$ flight variables monitored during a 70-meter flight over a flat terrain [1]. In Fig. 6A (left), the operator simply commanded the $\mathrm{MH}$ to pitch forward rampwise by an angle $\Delta \Theta=+10^{\circ}$ (between arrowheads 1 and 2). The ensuing increase in groundspeed $\boldsymbol{V}_{\boldsymbol{x}}$ (which reached $3 \mathrm{~m} / \mathrm{s}$, see B) automatically made the $\mathrm{MH}$ take off, since the feedback loop consistently increased $\boldsymbol{h}$ proportionally to $V_{\boldsymbol{x}}$ to comply with Eq 2. 
The MH eventually flew level at a groundheight $\boldsymbol{h}$ of approximately 1 meter - the value imposed by the OF setpoint $\omega_{\text {set }}=172^{\circ} / \mathrm{s}$ (Fig. 6C). After covering 42 meters, the $\mathrm{MH}$ was commanded to pitch backwards rampwise by an opposite angle $\Delta \Theta=-10^{\circ}$ (between arrowheads 3 and 4), and the ensuing deceleration (see B) automatically induced a gradual descent. The final approach (starting at arrowhead 4, Fig. 6A) was made at a constant descent angle $\boldsymbol{\alpha}$, as actually observed in landing bees [10] and predicted by the OF regulator scheme [1].

The MH flight pattern shows how an airborne vehicle can take off, navigate and even land on flat terrain without having to measure any groundheights or groundspeeds, provided it is equipped with an $O F$ sensor facing the ground and an $O F$ regulator servoing the $\mathrm{OF}$ to a reference value. The $O F$ regulator concept and the robot's performances were found to account for a series of puzzling, seemingly unconnected flying abilities observed during the last 70 years in various species (fruitflies, honeybees, moths, mosquitoes, dung-beetles, migrating locusts and butterflies).
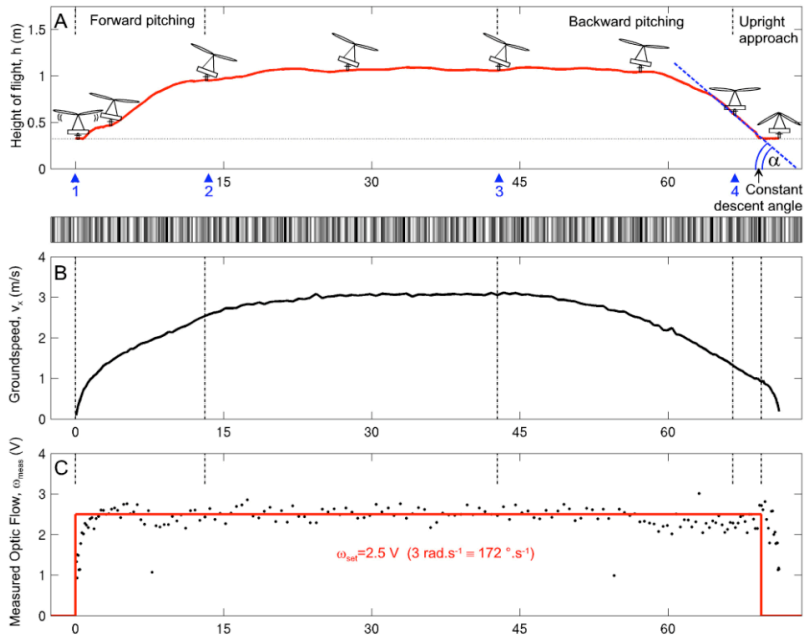

Figure 6: Groundheight (A), groundspeed (B) and optic flow (C) monitored during a 70-meter flight of the micro-helicopter $(\mathrm{MH})$ over the arena (the ground was flat here, without any rising slopes). The onboard OF regulator (Fig. 4A) suffices to make the robot perform a complete flight-path over the randomly textured pattern. Notice that the OF measured by the OF sensor (C) is held virtually constant (at $3 \mathrm{rad} / \mathrm{s}$, i.e., $172 \%$ s) throughout flight, including during take-off and landing where major changes in groundspeed occur (from [1]).

In line with the OCTAVE autopilot, we designed the LORA III autopilot (LORA III stands for Lateral Optic flow Regulator Autopilot, Mark III) [8]. LORA III is a dual OF regulator that is able to control both the forward speed $\boldsymbol{V}_{\boldsymbol{x}}$ of an aerial robot and its lateral distance to the right wall $\left(\boldsymbol{D}_{\boldsymbol{r}}\right)$ or left wall $\left(\boldsymbol{D}_{l}\right)$ in a corridor (Fig. 7).

We showed the feasibility of this scheme in simulation experiments where a miniature seeing hovercraft navigates in a straight or tapered corridor [8]. Our hovercraft is equipped with two additional lateral thrusters that make it fully actuated. It is therefore capable of independent side-slip and forward slip. LORA III is based on only two OF sensors (one looking to the right, one to the left). The groundspeed is constrained by

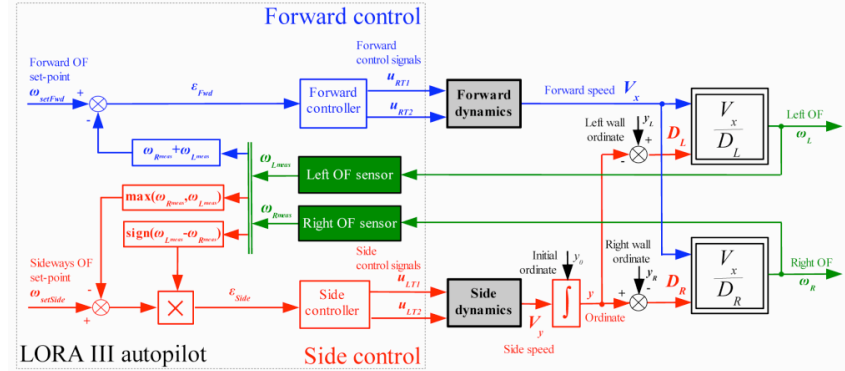

Figure 7: The LORA III autopilot is a dual OF regulator that enables a fully actuated hovercraft to navigate in a (straight or tapered) corridor by controlling its forward speed $\boldsymbol{V}_{x}$ and its distance $\boldsymbol{D}_{r}, \boldsymbol{D}_{\boldsymbol{l}}$ to the walls jointly, without requiring any speed or distance measurements (from [8]).

the environment, as observed on bees navigating in straight or tapered corridors [10]. The groundspeed $\boldsymbol{V}_{\boldsymbol{x}}$ is controlled by the error signal $\boldsymbol{\varepsilon}_{\boldsymbol{F} w \boldsymbol{w}}$ between the forward OF set point $\omega_{\text {setFwd }}$ and the sum of the two OFs (right and left). The clearance from the walls is constrained by the environment too: the lateral distance from one wall is controlled by the error signal $\boldsymbol{\varepsilon}_{\text {Side }}$ between the sideways OF set-point $\omega_{\text {setside }}$ and the larger of the two lateral OFs (right or left).
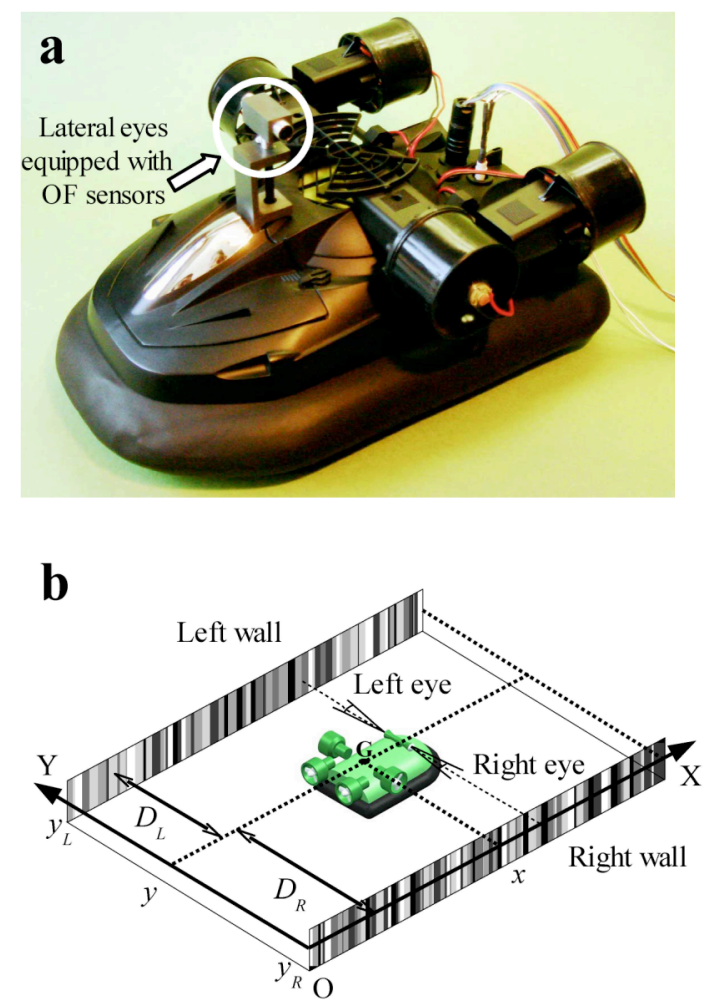

Figure 8: (a) Fully actuated hovercraft (HO) developed to test the LORA III autopilot. The HO $(36 \times 21 \times 14 \mathrm{~cm})$ is equipped with two eyes looking at an angle of $+90 /-90^{\circ}$ to the side. Each eye comprises only 2 pixels driving a single OF sensor (Fig. 2). (b) The walls of the corridor are randomly textured in both contrast and spatial frequency. The HO maintains its heading along the $\mathrm{X}$-axis via an inertial heading lock system actuating the rear thrusters differentially. The $O F$ experienced by each eye is proportional to the groundspeed and inversely proportional to the distances from the wall (from [8]). 
The advantage of this control scheme is that it determines both the forward speed and the distance from the walls on the sole basis of two constants - two OF setpoints - without any needs for measuring forward speed, lateral distances and corridor width, that is, without any needs for onboard velocimeters and rangefinders whatsoever. Extensive simulations experiments were presented, showing how the robot copes with major OF perturbations brought about by, e.g., an opening in one wall, a moving wall or a tapered corridor [8].

The LORA III dual OF regulator accounts particularly well for two types of behavior observed on bees flying freely in a corridor: the "centering behavior" $[4,10]$ and the "wall-following behavior" [9]. We showed that a "centering behavior" will simply ensue from a more general "wall-following behavior" whenever the values of the OF set-points $\boldsymbol{\omega}_{\text {setFwd }}$ and $\omega_{\text {setSide }}$ meet particular conditions with respect to each other [8].

\section{CONCLUSION}

OCTAVE and LORA III autopilots consist of feedback control loops called "optic flow regulators", the block diagrams of which (Fig. 4, 7) show which variables are measured, which ones are controlled, which ones are regulated (i.e., maintained constant), while giving the causal and dynamical relationships between these variables. In contrast with conventional aircraft' autopilots, OCTAVE and LORA III control loops do not aim to achieve any "speed holding" or "distance holding" abilities. They aim instead to modulate the behavior by an "OF hold" process that does without any measurements of speed and range. This $O F$ regulator process consistently tunes the animal behavior so as to make the OFs deviate little from the OF set-points, and therefore greatly reduces the dynamic range constraints imposed upon the OF sensors themselves [1].

- From a biological viewpoint, these explicit control schemes are interesting working hypotheses because they account for a number of puzzling, seemingly unconnected flight behaviors observed in many insect species over the last 70 years - including terrain following, sensible reactions to headwind, flight over mirror-smooth water, flight along tapered corridors, and landing at a constant slope on flat surfaces, as discussed in details in $[1,3]$. Our novel finding that bees do not center systematically in a corridor and tend to follow a wall [9] cannot be accounted for by the "optic flow balance hypothesis" $[4,10]$ but is well accounted for by the LORA III model, where "centering behavior" arises as a particular case of "wall-following behavior" [8]. It should be added that the neural implementation of an $\mathrm{OF}$ regulator is undemanding since it requires only a few linear operations (such as adding, subtracting an applying various filters) and nonlinear operations (such as minimum and maximum detections).

- From an engineering viewpoint too, these OF regulation schemes are undemanding and attractive since they do not rely on any rangefinders or velocimeters and can therefore do without the bulky and power-hungry emissive sensors of conventional avionics, such as Radars, Ladars or Flirs that equip many aircraft and spacecraft. Once engineered beyond the state of the minimalistic demonstrators presented here, OCTAVE and LORA III principles could potentially be harnessed to provide micro aerial, underwater and space vehicles with a certain degree of autonomy when they are to navigate in uncertain closed quarters or through complex terrains such as mountainous canyons.

\section{ACKNOWLEDGEMENTS}

We are grateful to S. Viollet, F. Aubépart, L. Kerhuel and G. Portelli for their fruitful comments and suggestions during this research. We are indebt to $\mathrm{M}$. Boyron, Y. Luparini and F. Paganucci for their expert technical assitance in electronics or micromechanics. This research was supported by CNRS (Life Science, Information and Engineering Science), an EU contract (IST/FET- 1999-29043) and a DGA contract (20050451037).

\section{REFERENCES}

[1] N. Franceschini, F. Ruffier and J. Serres. A bio-inspired flying robot sheds light on insect piloting abilities. Current Biol. 17: 329-335, 2007 [2] N. Franceschini, F. Ruffier and J. Serres.

Insects autopilots : Obstacle avoidance and speed control in insects and micro-aerial vehicles. In: Flying Insects and Robots (Floreano, D. et al., Eds.). Chapter 3, Springer, Berlin, 2009, in press

[3] N. Franceschini, F. Ruffier, J. Serres and S. Viollet. Optic flow based visual guidance: from flying insects to miniature aerial vehicles. In: Aerial Vehicles, (T.M. Lam, Ed.), Chapt. 35. In-tech Publ., Vienna, Austria, pp. 747-770, 2009

[4] Kirchner, W.H. ; Srinivasan, M.V. Freely moving honeybees use image motion to estimate distance. Naturwissenschaften, 76: 281-282 (1989)

[5] T. Netter and N. Franceschini. Neuromorphic optical flow sensing for Nap-of-the-Earth flight. In : Mobile Robots XIV, D.W. Gage and H.M. Choset (Eds), SPIE, Vol. 3838, pp. 208-21,1999

[6] T. Netter and N. Franceschini. A robotic aircraft that follows terrain using a neuromorphic eye. In: Proc. IEEE Intern. Conf. Intelligent Robots \& Systems (IROS02) Lausanne, Switzerland, pp. 129-134, 2002 [7] F. Ruffier, F., N. Franceschini. OCTAVE, a bioinspired visuomotor control system for the guidance of micro-aircraft. In: Bioengineered and Bioinspired Systems, SPIE Vol. 5119, Rodriguez A., D. Abbott, R. Carmona (Eds), Bellingham, USA, pp. 1-12, 2003

[8] J. Serres, D. Dray, F. Ruffier, N. Franceschini. A vision-based autopilot for a miniature air vehicle: joint speed control and lateral obstacle avoidance. Autonomous Robots 25: 103-122, 2008

[9] J. Serres, G. Masson, F. Ruffier and N. Franceschini.

A bee in the corridor : centering and wall-following.

Naturwissenschaften, 95: 1181-1187, 2008

[10] MV. Srinivasan, SW. Zhang, M. Lehrer, T. Collett. Honeybee navigation en route to the goal: visual flight control and odometry. J. Exp. Biol. 199: 237-244,1996

[11] MV. Srinivasan, SW. Zhang, J. Chahl, F. Barth, S. Venkatesh. How honeybees make grazing landing on flat surfaces. Biological Cybernetics 83: 171-183, 2000 